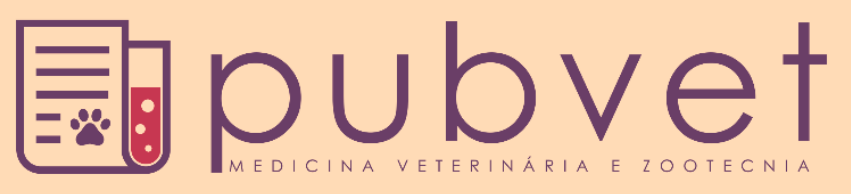

https://doi.org/10.31533/pubvet.v14n7a601.1-7

\title{
O cão não é o vilão: Vamos falar sobre leishmaniose?
}

\author{
Anita de Souza Silva ${ }^{1 *}{ }^{\circ}$, Renata Rocha da $\operatorname{Silva}^{2}{ }^{\circ}$, Barbara Regina Marques $^{3}{ }^{\circ}$, Brenda \\ Alessandra Santos Silva ${ }^{3}{ }^{\circ}$, Joyce Filho Santana ${ }^{3}$, Jhunya Francine de Melo Rocha ${ }^{4} \bullet$, Ylka \\ Priscilla Alves dos $\operatorname{Santos}^{1}{ }^{\ominus}$, Maria Josineide de Almeida ${ }^{\circ}$, Rogéria Pereira Souza $^{1}{ }^{\ominus}$, Roseane \\ Nunes de Santana Campos ${ }^{5}{ }^{\circ}$
}

${ }^{1}$ Discente do curso de Medicina Veterinária da Universidade Federal de Sergipe - Campus do Sertão. Nossa Senhora da Glória - SE Brasil. ${ }^{2}$ Discente do Programa de Pós-graduação em Ciências da Saúde, Universidade Federal de Sergipe. Aracaju - SE Brasil.

${ }^{3}$ Médico Veterinário pela Universidade Federal de Sergipe - Campus do Sertão. Nossa Senhora da Glória - SE Brasil.

${ }^{4}$ Discente do curso de Medicina Veterinária da Universidade Federal de Sergipe. São Cristóvão - SE Brasil.

${ }^{5}$ Docente do Núcleo de Medicina Veterinária da Universidade Federal de Sergipe - Campus do Sertão. Nossa Senhora da Glória - SE Brasil.

*Autor para correspondência, E-mail: $\underline{\text { anitasouza581@gmail.com }}$

Resumo. Atualmente, os animais de estimação são considerados membros da família, a convivência com estes proporciona inúmeros benefícios aos seres humanos, porém se os tutores de cães e gatos não tiverem informações adequadas sobre tutoria responsável, estes podem estar propensos aos maus tratos, abandono e a zoonoses. A Leishmaniose visceral canina (LVC) no Brasil é uma zoonose comumente causada pelo protozoário Leishmania infantum é transmitida através do repasto sanguíneo de fêmeas de flebótomos do gênero Lutzomia spp. Esta doença apresenta uma alta mortalidade em humanos e cães, sendo considerada uma patologia de relevante importância para a saúde pública. Em regiões do Nordeste brasileiro é observada uma grande quantidade de animais não domiciliados, além disso, a leishmaniose visceral é endêmica em cães e humanos nessa região, por isso as ações desenvolvidas nesse trabalho tiveram como objetivo conscientizar tutores de cães da região nordeste, especificamente do alto sertão sergipano, sobre a leishmaniose visceral demonstrando a etiologia, medidas de controle e prevenção da doença. Para isso, foram realizadas atividades em eventos importante do município, ações na Universidade Federal de Sergipe e visitas em escolas da região. Em média 200 adultos e 600 crianças foram informadas sobre as formas de controle e prevenção da enfermidade, além disso estas ações também tiveram como finalidade educar que não se adquire a doença através do contato direto com o cão. Este trabalho contribuiu com o processo de transformação nas atitudes da população local em relação às zoonoses, leishmaniose visceral e responsabilidade com os animais, sendo que esses temas são essenciais para melhorias do bem estar animal e da saúde pública.

Palavras chave: educação, leishmania spp., saúde pública, zoonoses

\section{The dog is not the villain: let's talk about leishmaniasis?}

Abstract. Nowadays, pets are considered members of the family, living with them provides numerous benefits to humans, however if dog and cat tutors do not have adequate information about responsible tutoring, these animals may be susceptible to abuse, abandonment and zoonoses. Canine visceral leishmaniasis (CVL) in Brazil is a zoonosis commonly caused by the protozoan Leishmania infantum and transmitted through the blood meal of female sandflies of the genus Lutzomia spp. This disease has a high mortality in humans and dogs, being considered a pathology of great importance for public health. In regions of North-eastern Brazil, a large number of non-domiciled animals are observed, in addition, visceral leishmaniasis is endemic in dogs and humans in this region, so the actions developed in this work aimed to raise awareness of dog tutors from the Northeast region, 
specifically from the high sergipano hinterland, about visceral leishmaniasis, demonstrating the etiology, measures of control and prevention of the disease. For this, activities were carried out at important events in the municipality, actions at the Federal University of Sergipe and visits to schools in the region. An average of 200 adults and 600 children were informed about ways to control and prevent the disease, in addition, these actions also aimed to educate that the disease is not transmitted through direct contact with the dog. This work contributed to the process of transforming the attitudes of the local population towards zoonosis, visceral leishmaniasis and responsibility towards animals, because these themes are essential for improving animal welfare and public health.

Keywords: education, leishmania spp., public health, zoonosis

\section{El perro no es el villano: ¿hablamos de leishmaniasis?}

Resumen. Actualmente, las mascotas se consideran miembros de la familia, vivir con ellas proporciona numerosos beneficios a los seres humanos, sin embargo, si los tutores de perros y gatos no tienen información adecuada sobre la tutoría responsable, pueden ser propensos a abusos, abandono y zoonosis. La leishmaniasis visceral canina (CVL) en Brasil es una zoonosis comúnmente causada por el protozoo Leishmania infantum que se transmite a través del repasto sanguíneo de las hembras del género Lutzomia spp. Esta enfermedad tiene una alta mortalidad en humanos y perros, siendo considerada una patología de importancia relevante para la salud pública. En las regiones del noreste de Brasil, se observa una gran cantidad de animales no domiciliados, además, la leishmaniasis visceral es endémica en perros y humanos en esta región, por esta razón, las acciones desarrolladas en este trabajo tenían como objetivo sensibilizar a los tutores de perros de la región noreste, específicamente del alto sertão sergipano, sobre la leishmaniasis visceral, demostrando la etiología, el control y las medidas de prevención de la enfermedad. Para ello, se realizaron actividades en eventos importantes en el municipio, acciones en la Universidad Federal de Sergipe y visitas a escuelas de la región. Se informó a un promedio de 200 adultos y 600 niños sobre las formas de controlar y prevenir la enfermedad, además, estas acciones también tenían como objetivo educar que la enfermedad no se adquiere a través del contacto directo con el perro. Este trabajo contribuyó al proceso de transformación de las actitudes de la población local hacia las zoonosis, la leishmaniasis visceral y la responsabilidad hacia los animales, y estos temas son esenciales para mejorar el bienestar animal y la salud pública.

Palabras clave: educación, leishmania spp., salud pública, zoonosis

\section{Introdução}

A Leishmaniose visceral (LV) no Brasil é considerada uma zoonose causada comumente pelo protozoário Leishmania infantum (Spada et al., 2016) e transmitida principalmente pelo repasto sanguíneo da fêmea do flebotomíneo Lutzomyia longipalpis, conhecido popularmente como mosquito palha ou birigui (Gontijo \& Melo, 2004; N. B. Moreira et al., 2016; Schimming \& Silva, 2012; Silva et al., 2005; Youssef et al., 2019). A leishmaniose é uma doença com alta letalidade, uma patologia de grande importância para saúde pública (Belo et al., 2013).

Os animais domésticos, principalmente o cão têm assumido um importante papel como reservatório da infecção no ciclo de transmissão da leishmaniose, devido ao intenso parasitismo cutâneo que este animal apresente após infecção (Youssef et al., 2019). Todavia, o contato com os animais infectados não oferece riscos aos seres humanos (Schimming \& Silva, 2012), já que a transmissão para o homem e para os hospedeiros mamíferos ocorre durante o repasto sanguíneo das fêmeas de flebótomos infectados, quando este regurgita formas infectantes do protozoário, promastigotas metacíclica, na derme dos hospedeiros vertebrados (van Griensven \& Diro, 2019).

$\mathrm{Na}$ maioria das áreas onde a doença é endêmica, o conhecimento sobre a leishmaniose visceral e formas de prevenção restringe-se, muitas vezes, às pessoas que tiveram a doença ou a casos na família, ou vizinhança (Uchôa et al., 2004; Youssef et al., 2019). Os trabalhos educativos com a comunidade 
auxiliam no controle de zoonoses, informações sobre tutoria responsável de cães e gatos e as formas de controle da leishmaniose visceral, evitam a disseminação dessa enfermidade (Broom, 1991; Marder et al., 2004; Molento, 2005; Souza et al., 2016).

Dessa forma, a ação de extensão intitulada "O cão não é o vilão: vamos falar sobre leishmaniose?" teve como objetivo conscientizar tutores de cães do alto sertão sergipano, sobre a leishmaniose visceral, com relação aos riscos, medidas de controle e prevenção da doença.

\section{Material e métodos}

\section{Descrição da área de estudo}

As ações foram realizadas no Município de Nossa Senhora da Glória, que se localiza na Região Nordeste do Brasil, no oeste do Estado de Sergipe, na microrregião do alto sertão sergipano. Apresenta uma estimativa de população de 36.924 habitantes (IBGE, 2019).

\section{Abordagem}

Para a execução das ações, inicialmente houve reunião com a coordenadora responsável e os discentes envolvidos no projeto de extensão para conversas e pesquisas sobre a leishmaniose visceral, abordando o ciclo de transmissão, formas de controle e prevenção da doença, além da confecção de folders informativos para os tutores de cães.

\section{Etapa 1. Expo Ouro Branco em Nossa Senhora da Glória, Sergipe.}

O projeto desenvolveu atividades com as crianças e adolescentes das escolas municipais e adultos da comunidade na exposição agropecuária Expo Ouro Branco. Esta exposição é um evento relevante no município, sendo que ocorre em três dias consecutivos. No local houve apresentação do ciclo de transmissão da leishmaniose visceral e formas de prevenção da doença, também houve sorteio de brindes como coleiras repelentes e entrega de folders explicativos (Figuras 1A e 1B).
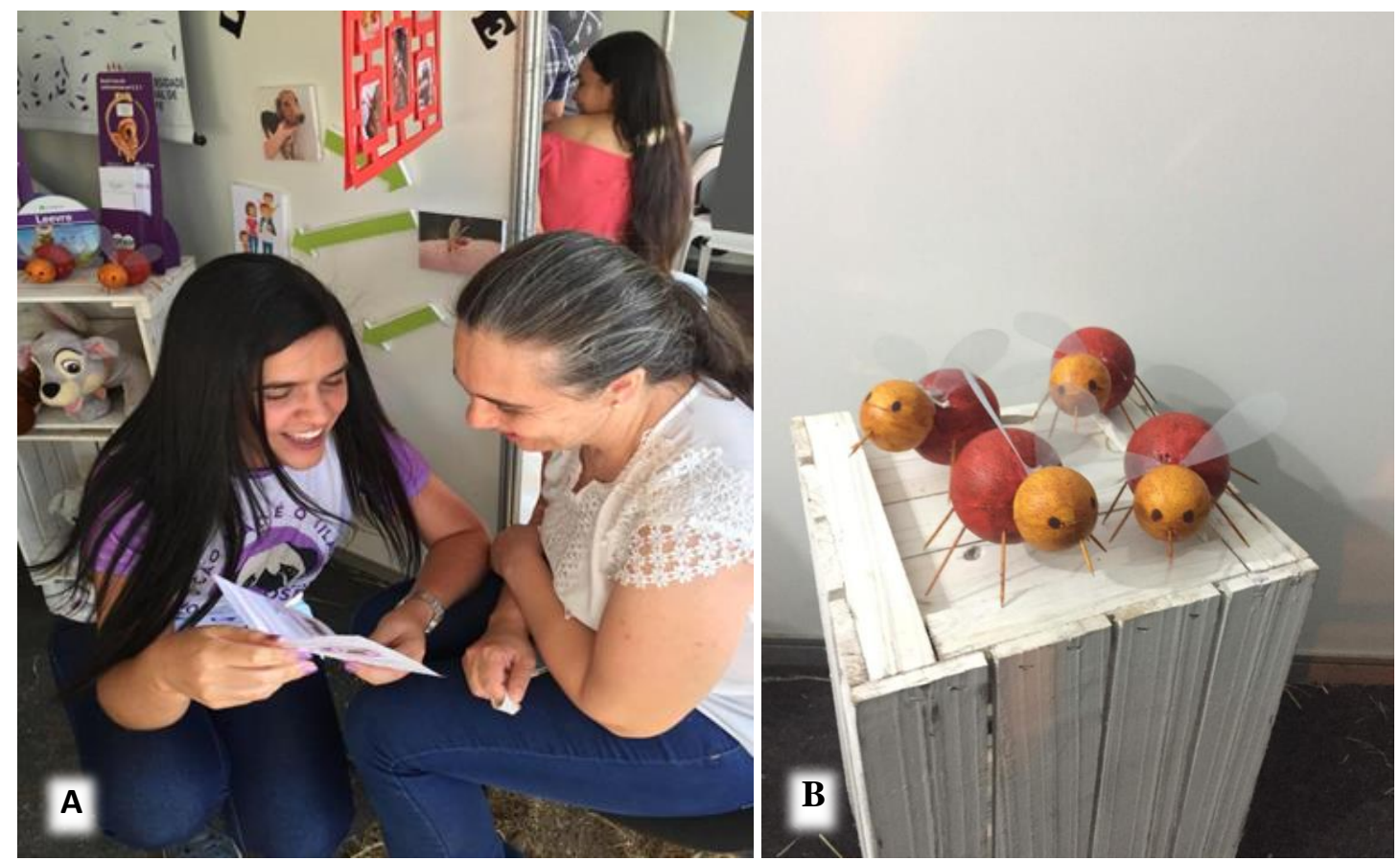

Figura 1. Expo Ouro Branco. (A). Folders explicativos aos tutores. (B). Flebótomos lúdicos expostos na exposição.

\section{Etapa 2. Ações na Universidade Federal de Sergipe}

As ações no Campus do sertão da Universidade Federal de Sergipe, aconteceram em dois eventos (Figuras 2A e 2B) realizados pelo núcleo de medicina veterinária, na qual os tutores eram orientados sobre a 
formas de prevenção da leishmaniose visceral e a entrega de folders que explicavam a etiologia, sinais clínicos e prevenção da leishmaniose visceral nos cães, foram realizados sorteios de coleira repelente.

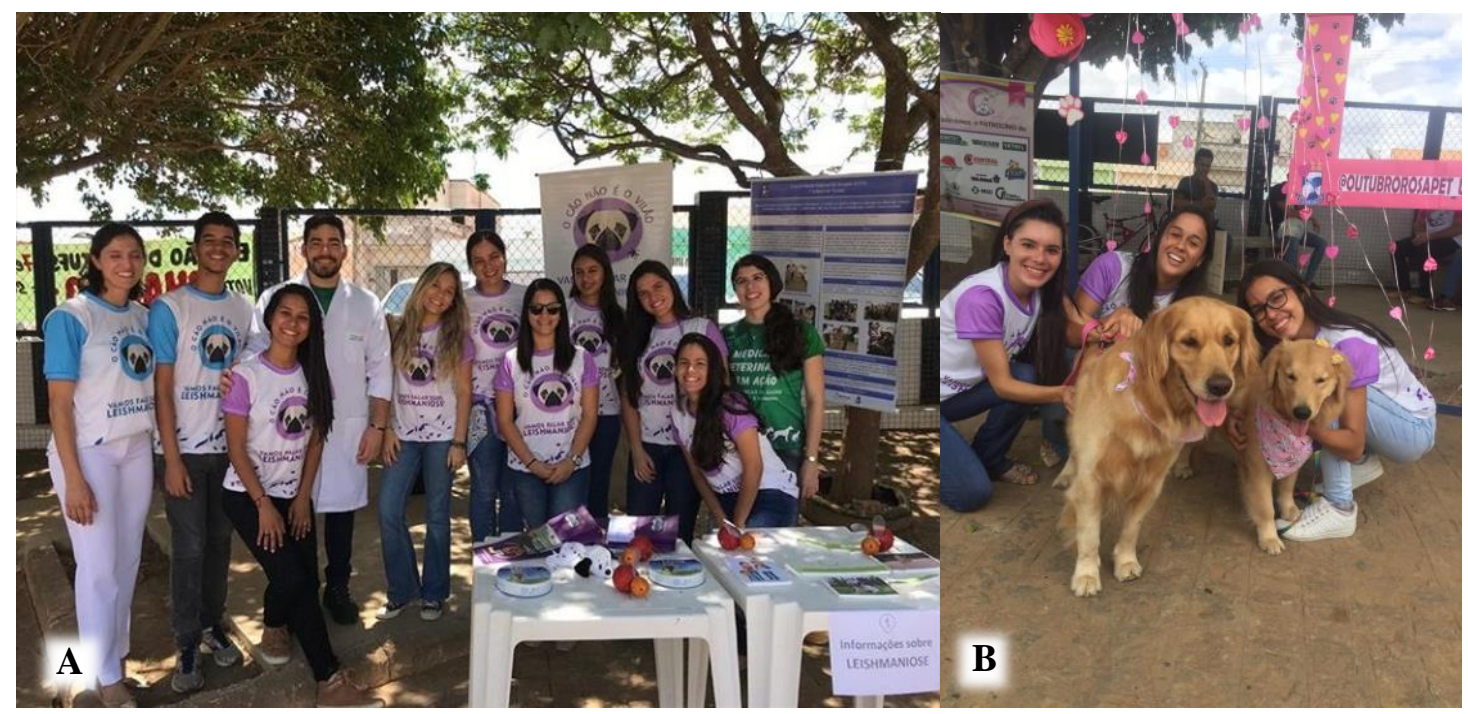

Figura 2. Eventos. (A). Dia D: Ação de extensão da UFS Campus Sertão. (B). I outubro Rosa Pet do Sertão.

Etapa 3. Ações em escolas da rede pública do município de Nossa Senhora da Glória, Sergipe.

Foram realizadas visitas em três escolas públicas da rede municipal de Nossa Senhora da Glória, Sergipe. Nestes locais eram desenvolvidas atividades lúdicas educativas, como: peças teatrais, brincadeiras, pinturas faciais e vídeos abordando temas como bem-estar animal e leishmaniose visceral, enfatizando através das atividades, como ocorre a transmissão da leishmaniose, o ciclo da doença e as formas de prevenção (Figuras 3A e 3B).
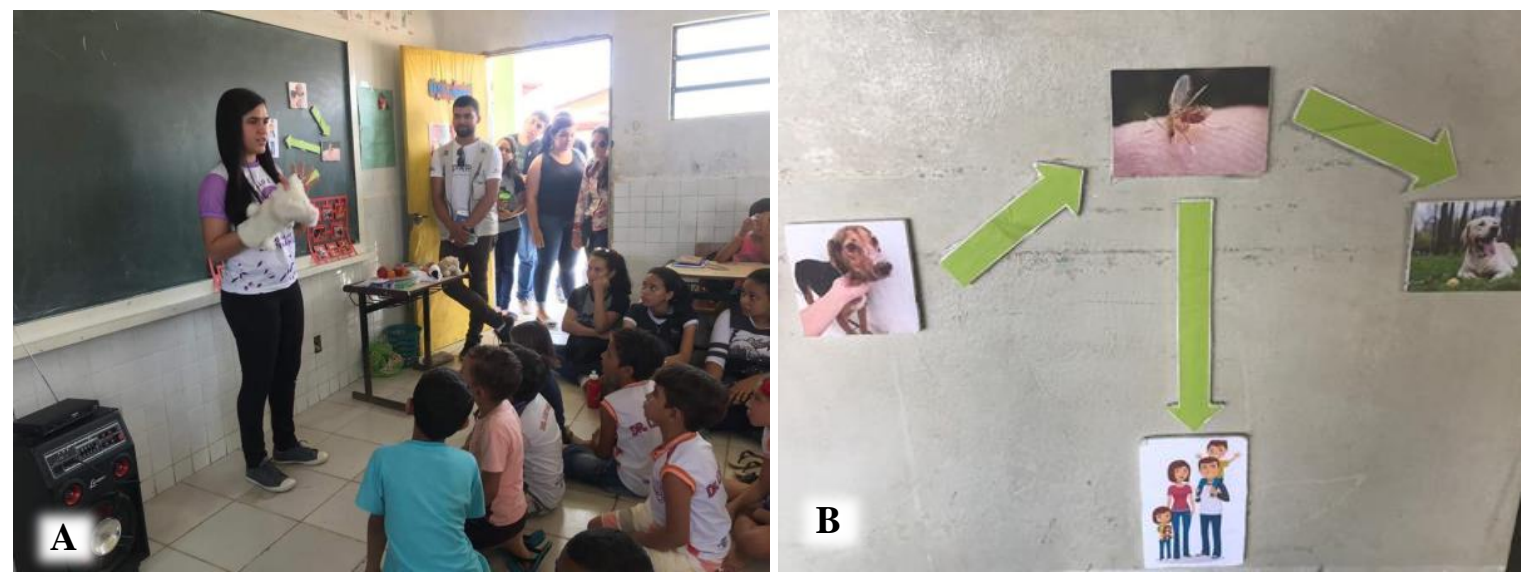

Figura 3. Atividades lúdicas. (A). Peça teatral. (B). Ciclo epidemiológico da leishmaniose visceral.

Ao final das ações havia perguntas e repostas sobre o conteúdo informado e todas as crianças recebiam brindes para estimular a participação. As crianças, os diretores, os professores e indiretamente, pais e responsáveis eram orientados sobre a leishmaniose visceral, pois eram entregues folders para que as crianças levassem para as residências e explicassem aos responsáveis o que foi informado sobre a doença na escola (Figura 4A).

\section{Resultados e discussão}

Ações de extensão desenvolvidas junto à comunidade são imprescindíveis para a promoção do conhecimento (Rodrigues et al., 2013). A eficiência da participação da população como agentes multiplicadores da informação em ações de prevenção e controle de doenças, inclusive da leishmaniose, já foi comprovada (Lobo et al., 2013; Youssef et al., 2019). 
Durante as ações realizadas houve a apresentação do ciclo de transmissão da leishmaniose visceral para tutores de cães e crianças, visto que foi demonstrado a falta de conhecimento da população referente a esta doença (Lobo et al., 2013; Lopes et al., 2019). Foi possível demonstrar que a transmissão para o ser humano não ocorre por meio de contato direto com o cão infectado, sendo transmitido apenas após o repasto sanguíneo da fêmea de insetos flebótomos infectados (Lara-Silva et al., 2015).
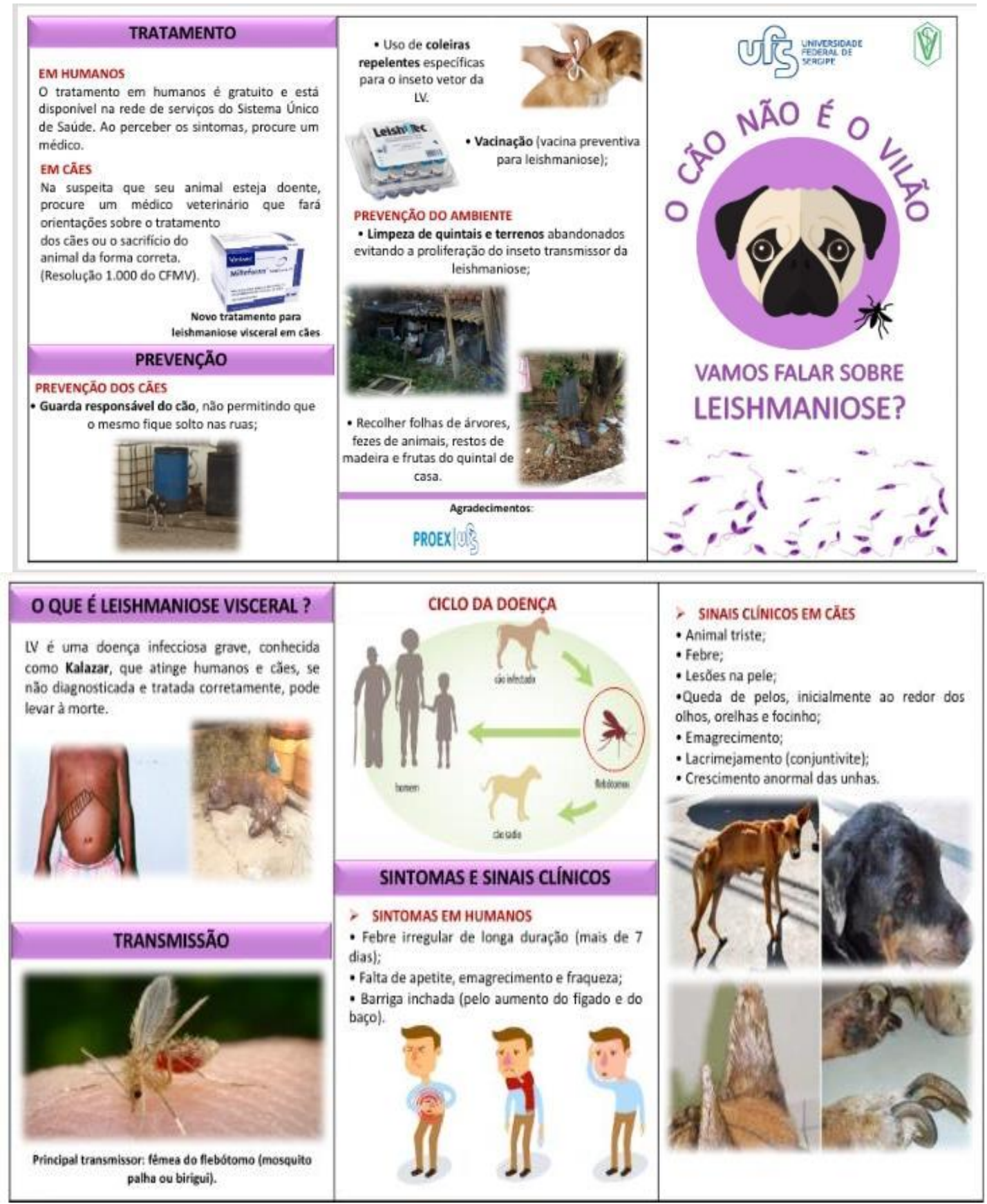

Figura 4. Folder elaborado pelos discentes contendo informações sobre a leishmaniose visceral.

As formas mais eficazes de evitar a doença são através do controle e prevenção (Abbiati et al., 2019). Estas medidas contribuem para a diminuição da incidência da doença (Lopes et al., 2019). Nas ações as formas de prevenção da leishmaniose foram ressaltadas. Houve orientação sobre a importância do uso de coleiras repelentes (Schimming \& Silva, 2012), cuidados de higiene, como evitar o acúmulo de lixo, visto que, o vetor costuma se reproduzir em locais que contenham matéria orgânica em decomposição (Costa, 2011), aplicação de inseticidas ambientais onde o animal permanece por mais tempo e evitar passeios crepusculares ou noturnos, já que são horários de maior atividade dos flebotomíneos (Schimming \& Silva, 2012), além do uso de vacinas na prevenção da doença nos cães (Moreira, 2013). 
Nas ações, foi perceptível que muitas pessoas conhecem somente a eutanásia de cães como forma de controle da doença, pois, durante décadas, as autoridades de saúde pública tentam reduzir a incidência de leishmaniose com o sacrifício em massa de cães infectados, porém essas atitudes não demonstraram sucesso na diminuição dos casos da doença em humanos (Dantas-Torres et al., 2020), por isso, durante os eventos eram abordadas estratégias de controle e prevenção da enfermidade e ressaltado que o contato direto com cão não transmite a doença, além disso, era informado a população sobre a importância da guarda responsável de animais de companhia.

As visitas em escolas públicas do município de Nossa Senhora da Glória, Sergipe propiciaram a sensibilização de 600 crianças e de forma transversal os pais de estudantes e colaboradores das escolas. Nas ações, foi possível observar que algumas crianças não conheciam a doença, dessa forma foram informadas sobre a etiologia, formas de transmissão e as medidas de controle da doença. Crianças em idade escolar, possuem grande capacidade de aprender conceitos abordados e compartilhá-los com pessoas próximas (Silveira et al., 2012). As atividades lúdicas são recursos bastante utilizados no aprendizado de crianças (Almeida et al., 2014). As crianças aprendiam sobre a leishmaniose visceral e tutoria responsável de animais através de uma forma interativa e disseminavam as informações na comunidade.

Além das atividades realizadas pelos discentes nos eventos que envolviam à população do município, foi entregue um folder informativo sobre a doença com o intuito de compartilhar o aprendizado, abrangendo mais pessoas.

Apesar do contato com os animais não oferecer riscos aos seres humanos, a maioria das doenças zoonóticas pode ser reduzida, ou até eliminada, mediante práticas de manejo adequada, políticas públicas e educação dos tutores sobre guarda responsável (Canatto et al., 2012).

\section{Conclusão}

Ações de educação em saúde proporcionam a comunidade informações importantes. Explicações sobre etiologia, ciclo de transmissão, formas de prevenção da leishmaniose visceral e tutoria responsável em animais de companhia promove melhorias no bem-estar animal, na saúde pública, prevenindo a disseminação de doenças, principalmente zoonoses.

\section{Agradecimentos:}

A Pró - Reitoria de Extensão da Universidade Federal de Sergipe (PROEX- UFS) e a Biomanguinhos - FIOCRUZ.

\section{Referências bibliográficas}

Abbiati, T. C., Freitas, D. M. de, Alves, L. C., Freitas, B. G., Rezende, R. S., Barbosa, S. G., Jorge, A. L. T. A., Santos, S. M. \& Lopes, M. C. (2019). Leishmaniose visceral canina: Relato de caso. PUBVET, 13, 1- 8. DOI: https://doi.org/10.31533/pubvet.v13n4a307.1-8

Almeida, J. F., Pedro, D. A., Pereira, V. L. A., Abreu, D. L. da C. \& Nascimento, E. R. (2014). Educação humanitária para o bem-estar de animais de companhia. Enciclopédia Biosfera, 10, 1366-74.

Belo, V. S., Struchiner, C. J., Werneck, G. L., Barbosa, D.S., Oliveira, R. B., Neto, R. G. T. \& Silva, E S. (2013). A systematic review and meta-analysis of the factors associated with Leishmania infantum infection in dogs in Brazil. Veterinary Parasitology, 195(1-2),1-13. DOI: https://doi.org/10.1016/j.vetpar.2013.03.010

Broom, D. M. (2005). Animal welfare education: development ad prospects. Journal of Veterinary Medical Education, 32, 438-44. DOI: https://doi.org/10.3138/jvme.32.4.438

Canatto, B. D., Silva, E. A., Bernardi, F., Mendes, M. C. N. C., Paranhos, N. T. \& Dias, R. A. (2012). Caracterização demográfica das populações de cães e gatos supervisionados do município de São Paulo. Arquivo Brasileiro de Medicina Veterinária e Zootecnia, 64, 1515-1523. DOI: https://doi.org/10.1590/S0102-09352012000600017

Costa, C. H. N. (2011). How effective is dog culling in controlling zoonotic visceral leishamniasis? A critical evaluation of the science, politics and ethics behind this public health policy. Revista da Sociedade Brasileira de Medicina Tropical, 44, 232-242. DOI: https://doi.org/10.1590/S003786822011005000014 
Dantas-Torres, F., dos Santos Nogueira, F., Menz, I., Tabanez, P., Silva, S. M., Ribeiro, V. M., Miró, G., Cardoso, L., Petersen, C., Baneth, G., Oliva, G., Solano-Gallego, L., Ferrer, L., Pennisi, M. G., Bourdeau, P., Maia, C., Otranto, D., Gradoni, L., Courtenay, O. \& Costa, C. H. N. (2020). Vaccination against canine leishmaniasis in brazil, International Journal for Parasitology, 50, 171-176.

Instituto Brasileiro de Geografia e Estatística. (2019). Estimativas da população residente para os municípios e para as unidades da federação com data de referência em $1^{\circ}$ de julho de 2019: [notas metodológicas] Rio de Janeiro: IBGE.

Lara-Silva F. D. O., Michalsky, É. M., Fortes-Dias, C. L., Fiuza, V. D. O. P., Pessanha, J. E. M. \& Regina-Silva S. (2015). Epidemiological aspects of vector, parasite, and domestic reservoir in areas of recent transmission and no reported human cases of visceral leishmaniasis in Brazil. Acta Trop, 148: 128-36. DOI: https://doi.org/10.1016/j.actatropica.2015.04.002

Lobo, K. dos S., Bezerra, J. M. T., Brito, L. M. de O, Silva, J. S. \& Pinheiro. V. C. S. (2013). Conhecimentos de estudantes sobre Leishmaniose Visceral em escolas públicas de Caxias, Maranhão, Brasil. Ciênc. saúde coletiva, 18, 2295-2300.

Lopes, G. S., Sousa, V. A., Martins, J. S. C., Sousa, E. S. \& Cajaiba, R. L. (2019). Nível de conhecimento e medidas de prevenção de moradores sobre a Leishmaniose Visceral em área endêmica no Maranhão, Brasil. Archives of Health Investigation, 8, 285- 289. DOI: https://doi.org/10.21270/archi.v8i6.3239

Marder, A. (2015). Intake and assessment. In: Animal behavior for shelter veterinarians and staff. Weiss, E., Mohan-Gibbons, H. \& Zawistowski, S. Wiley Blackwell, 386.

Molento, C. F. M. (2005). Bem-estar e produção animal: aspectos econômicos - revisão (Animal) welfare and production: economic aspects - Review), Archives of Veterinary Science, 10, 1-11.

Moreira, M. L. (2013). Duração da imunidade vacinal da leishmaniose visceral canina: perfil fenotípico e funcional da atividade fagocítica da anti-Leishmania chagasi. [M.S. dissertation], Ministry of Health, Oswaldo Cruz Foundation, Rio de Janeiro, Brasil.

Pace, D. (2014). Leishmaniasis. Journal of Infection, 69, 10-18.

Rodrigues, A. L. L., Prata, M. S., Batalha, T. B. S., Costa, C. L. N. A. \& Neto, I. F.P. (2013). Contribuições da extensão universitária na sociedade. Cadernos de Graduação - Ciências Humanas e Sociais, 1, 141-148.

Schimming, B. C. \& Pinto e Silva, J. R. C. (2012). Leishmaniose Visceral Canina - Revisão de literatura. Revista Científica Eletrônica De Medicina Veterinária, 19, 1-17.

Silva, A. V. M., Paula, A. A., Cabrera, M. A. A. \& Carreira, J. C. A. (2005). Leishmaniose em cães domésticos: aspectos epidemiológicos. Caderno de Saúde Pública, 21, 324-332.

Silveira, C. A., Muzzi, L. C. C., Ferreira, F. de O., Slywitch, B. N. \& Alves, M. V. S. (2012). Conscientização sobre a posse responsável de animais domésticos em bairros e escolas do município de Uberlândia/MG, Revista Em Extensão, 11, 110-118. DOI: https://doi.org/10.14393/REE

Souza, A. F., Cruz, A. I. S., Rique, A. S., Brilhante, A. J. V. C., Farias, B. R. T., Rocha, J. J. G. \& Silva, S. V. (2016). O despertar da posse responsável na infância: saúde pública e cidadania. Revista Ciência e Extensão, 12, 29-40.

Uchôa, C. M. A., Serra, C. M. B., Magalhães, C. M., Silva, R. M. M., Figliuolo, L. P., Leal, C. A. \& Madeira, M. F. (2004). Educação em saúde: ensinando sobre a leishmaniose tegumentar americana. Caderno de Saúde Pública, 20(4):935-41.

Van Griensven, J. \& Diro, E. (2019). Visceral Leishmaniasis: Recent Advances in Diagnostics and Treatment Regimens. Infectious Disease Clinics of North America, 33, 79-99. DOI: https://doi.org/10.1016/j.idc.2018.10.005

Youssef, A. G., Netto, L. L., Tuani, B. R. V., Serafim, J. M. P., Teixeira, D. de B., Polegato, E. P. dos S., Porto, C. D. \& Friolani, M. (2019). Conhecimento populacional sobre leishmaniose no município de Marília, São Paulo, Brasil. Revista Acadêmica Ciência Animal, 17, 1-7. DOI: 10.7213/19814178.2019.17008

Recebido: 5 de maio, 2020.

Aprovado: 4 de junho, 2010.

Disponível online: 21 julho, 2020.
Licenciamento: Este artigo é publicado na modalidade Acesso Aberto sob a licença Creative Commons Atribuição 4.0 (CC-BY 4.0), a qual permite uso irrestrito, distribuição, reprodução em qualquer meio, desde que o autor e a fonte sejam devidamente creditados. 